\title{
Fully-coupled electromechanical simulations of the LV dog anatomy using HPC: Model testing and verification
}

\author{
Jazmin Aguado-Sierra $^{1 \star}$, Alfonso Santiago ${ }^{1}$, Matias I. Rivero ${ }^{1}$, Mariña \\ Lopez-Yunta $^{1}$, David Soto-Iglesias ${ }^{2}$, Lydia Dux-Santoy ${ }^{2}$, Oscar Camara ${ }^{2}$, and \\ Mariano Vazquez ${ }^{1}$ \\ 1 Barcelona Supercomputing Centre, Barcelona Spain, \\ 2 PhySense, DTIC, Universitat Pompeu Fabra, Barcelona, Spain
}

\begin{abstract}
Verification of electro-mechanic models of the heart require a good amount of reliable, high resolution, thorough in-vivo measurements. The detail of the mathematical models used to create simulations of the heart beat vary greatly. Generally, the objective of the simulation determines the modeling approach. However, it is important to exactly quantify the amount of error between the various approaches that can be used to simulate a heart beat by comparing them to ground truth data. The more detailed the model is, the more computing power it requires, we therefore employ a high-performance computing solver throughout this study. We aim to compare models to data measured experimentally to identify the effect of using a mathematical model of fibre orientation versus the measured fibre orientations using DT-MRI. We also use simultaneous endocardial stimuli vs an instantaneous myocardial stimulation to trigger the mechanic contraction. Our results show that synchronisation of the electrical and mechanical events in the heart beat are necessary to create a physiological timing of hemodynamic events. Synchronous activation of all of the myocardium provides an unrealistic timing of hemodynamic events in the cardiac cycle. Results also show the need of establishing a protocol to quantify the zero-pressure configuration of the left ventricular geometry to initiate the simulation protocol; however, the predicted zero-pressure configuration of the same geometry was different, depending on the origin of the fibre field employed.
\end{abstract}

Key words: High-Performance Computing (HPC), electromechanical simulations, canine model, ground-truth data, verification

\section{Introduction}

Biophysical models of the heart have significantly improved their realism during the last years by incorporating patient-specific geometries and boundary conditions obtained from processing multimodal images and signals. In addition, continuous improvements in hardware and infrastructure computing facilities, specially in High-Performance Computing (HPC) and Graphical Processing Units

\footnotetext{
* jazmin.aguado@bsc.es
} 
(GPU) are dramatically reducing computational costs associated to these tools. These are steps required for translating these biophysical models into clinical routine and develop simulation-based pipelines that can help on the management of a patient.

Nevertheless, electromechanical simulations of the heart remain a computational challenge extremely difficult to properly validate and verify. This is due to the complexity of the heart's physiology where multiple physical phenomena are tighly coupled at a different spatial and temporal scales, hampering the acquisition of complete sets of measurements to be used as reliable ground-truth data. Furthermore, detailed biophysical models including different spatial scales have a large amount of parameters that often are tuned based on limited experiments. Therefore, substantial effort is still required on performing sensitivity analysis of these parameters, verification studies to assess the appropriateness of the developed solvers and validation experiments to compare simulations with observations.

The majority of biophysical models of the heart were usually tuned to replicate observations obtained in laboratory experiments (e.g. patch clamp) that allowed to estimate some parameters. More recently some pioneering modelling work aimed at using patient-specific heart geometries derived from imaging and estimated some parameters comparing clinical measurements with electromechanical simulations [1-3]. Nevertheless, clinical measurements from humans are often incomplete and sparse, then not quite appropriate for exhaustive validation and verification of the electromechanical solvers. More controlled gold-standard electrophysiological data, derived either synthetically [4] or with experimental models [5], has been used in some simulation benchmarks to verify, customize, validate and integrate different cardiac electrophysiological solvers. A challenge organized in STACOM'11 aiming at validating myocardial tracking and deformation algorithms applied to image sequences [6], but, to our knowledge, there has not been yet a challenge for assessing simulated deformation fields provided by electromechanical models of the heart.

The STACOM'14 workshop included a challenge precisely aiming at evaluating electromechanical solvers of the heart using ground-truth data obtained from tag-MRI of experimental dog models. In this paper we make use of this data to test and verify the electromechanical simulations that have been developed over the last years at the anonymized centre. These simulations have been implemented to make use in the most efficient way high-performance computational resources, with a massively parallel implementation, an explicit formulation and a tight coupling between the electrical and mechanical solvers.

\section{Methods}

The software used for this study is an in-house, finite-element, multi-physics, High-Performance Computing (HPC) software. The experimental data, which is fully described described in [7], was kindly provided by the University of Auckland as part of the mechanical challenge at the STACOM'14 workshop. 


\subsection{Use of experimental data}

High resolution Magnetic Resonance Imaging (MRI) data from one of the four datasets of normal dogs were used. The point clouds defining the LV geometry at diastasis, were used to generate the mesh employed as the starting point of our simulation protocol. Two more left ventricular geometries were provided for each dog at two hallmark times of the cardiac cycle: end of filling (end-diastole) and end of contraction (end-systole). Left ventricular volume and pressure were measured experimentally at several time points when the animals were being paced at $500 \mathrm{~ms}$. The hemodynamic information was used to setup the appropriate boundary conditions during the cardiac cycle. They were also used to approximately tune some electric propagation information like total activation time, and action potential duration/repolarization.

\subsection{Mathematical description of the anatomy}

The provided endocardial and epicardial point clouds at diastasis were used to generate the mesh. Two meshes of different resolution were built using an inhouse volume mesh generation and visualization code called Iris. One of the resulting left ventricular mesh has 19,591 regular tetrahedral elements $(4,835$ nodes). The average element volume is $2.7 e-03 \mathrm{~cm}^{3}$, so that the average side lengths of the elements are $0.2874 \mathrm{~cm}$. The second left ventricular mesh has 226,079 regular tetrahedral elements (46, 401 nodes). The average element volume is $0.191204 e-03 \mathrm{~cm}^{3}$, so that the average side lengths of the elements are $0.11 \mathrm{~cm}$. Note that the framework used in this work uses CGS system of units (centimetre-gram-second). Note that the same mesh is used to solve both electrophysiology and biomechanics problems. Material parameter calibration was performed in the lowest resolution mesh.

\section{3 fibre Orientation Description}

Muscle fibre orientations were derived from ex vivo diffusion tensor MRI available from the dog experiments. They were provided as raw DTI data regularly sampled at a grid of points, already been registered to the in-vivo geometry in diastasis, and as fibres post-processed and interpolated at the nodes of an hexahedral mesh of 9,225 nodes. As part of our protocol, we generated a field of fibres as described by Streeter [8] at \pm 60 degrees to compare and analyse the impact on the electromechanics given the use of different fibre fields: Streeter and the given DTI.

\subsection{Electromechanical framework}

No measurements were provided from the dog experiments to adapt the electrical part of the solver, therefore two different assumptions on the behaviour of the left ventricle of a healthy dog were made to simulate electrical wave propagation: 

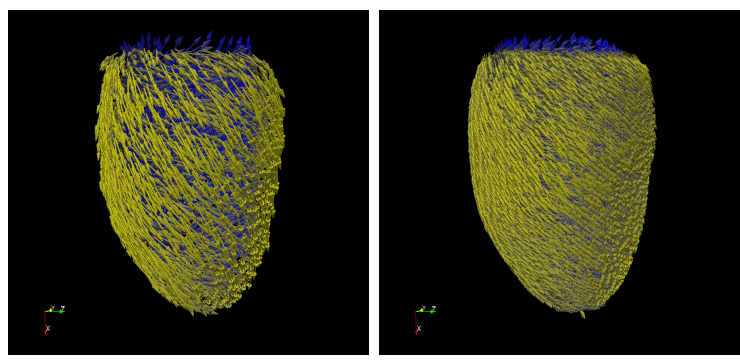

Fig. 1. Left: Synthetic fibre orientation generated using the model of Streeter et al. Right: Post-processed DTI fibres (at 9925 points) and interpolated into the high resolution, tetrahedral mesh using a closest neighbor interpolation.

- All of the myocardium was stimulated at the same time. This assumption holds if we assume a dense Purkinje system, an infinite conductivity and a synchronous activation of the whole myocardium.

- The activation occurs in the endocardium at the same time. This assumption holds if we assume a dense Purkinje fibre system. Conductivity was approximated by the knowledge of a "healthy, normal" ECG of a dog paced at a basic cycle length of $500 \mathrm{~ms}$.

Electrophysiology Ion concentration gradients across the myocyte cell membrane determine the electrical action potential that triggers contraction. To simulate the electrical activity, we solve a reaction-diffusion equation in our finite element mesh. We employ the monodomain formulation, solved explicitly [9]. The tissue is considered anisotropic to the fibre orientation. For this paper, we have employed the ionic cell models by Fitzhugh [10]. Conductivity in the fibre direction for the Fitzhugh-Nagumo model is $0.007 \mathrm{mS} / \mathrm{cm}$ in the fibre direction and $0.0023 \mathrm{mS} / \mathrm{cm}$ in the transverse directions. The conductivity was setup so that the total activation time occurs within $50 \mathrm{~ms}$.

\subsection{Biomechanics}

The mechanical deformation part is solved at the same resolution of the electrophysiology mesh, thus avoiding any type of errors induced by interpolation between different meshes used for different physical solvers. The myocardial constitutive model employed in this study is a transversally isotropic version of the Holzapfel and Ogden [12] model. Therefore, the myocardium is considered as a non-homogeneous, non-linearly elastic, slightly compressible material [13].

In this study, the phase of diastasis can be considered to be a stress-free state. However, pressure in diastasis is low, but not zero. To obtain accurate results in the computational simulations, the zero-pressure configuration must be the starting point of the simulations. A deflation protocol was established, to obtain the stressed geometry at the time of diastasis. 
Zero-pressure configuration protocol The first step is to "collapse" the geometry to a zero pressure state, in which the internal stresses of the solid can be assumed to be zero. The measured pressure in diastasis for the data in this simulation was $0.36 \mathrm{kPa}$. To deflate the geometry, we applied an equal, but opposite pressure to the endocardial walls of the left ventricular mesh. The resulting configuration is shown in figure 2 . The mesh was allowed to deflate until a steady state was reached, at a $V_{0}$ of $16.3 \mathrm{ml}$ for the DTI fibre mesh. Instead, for the mesh with the Streeter fibres, the $V_{0}$ was $16.7 \mathrm{ml}$. When volume was constant for each of the simulations, we obtained the geometry and fibre orientations in the assumed zero-pressure configuration and re-inflated the geometry to the pressure in diastasis. This process is iteratively done to recover the geometry in diastasis and is also used to calibrate the passive material parameters to obtain the pressure-volume relationship at the time of end diastole.
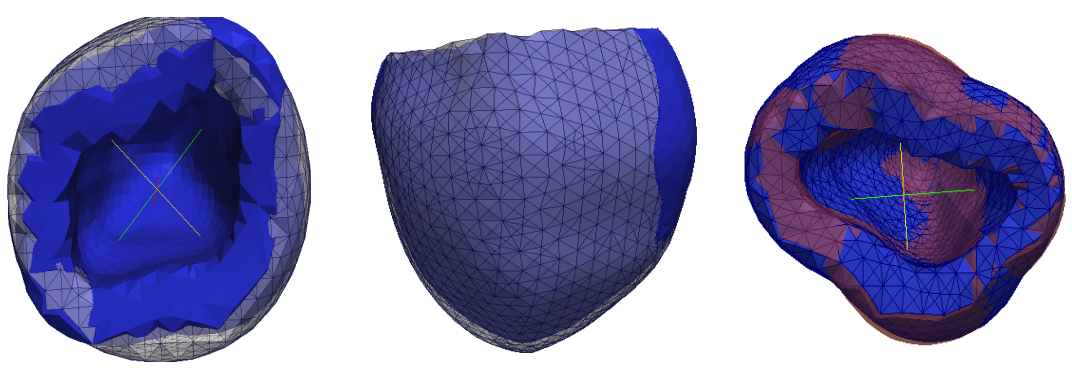

Fig. 2. Zero-pressure configuration of the LV geometry with DTI fibres in blue, compared to the initial geometry in white. Right: Zero pressure configuration of the DTI fibre mesh in blue; Streeter fibre mesh is shown in red.

\subsection{Excitation Contraction Coupling}

The electrophysiology model triggers the myocardial contraction using the model published by Hunter et al.[14], where a synthetic calcium transient is generated for the Fitlzhugh-Nagumo electrophysiology model as:

$$
C a_{i}(t)=C a_{0}+\left(C a_{\max }-C a_{0}\right) \frac{t}{\tau_{C a}} e^{1-t / \tau_{C a}}
$$

Where the parameters were modified to represent the calcium transient of a dog heart beat at a $500 \mathrm{~ms}$ basic cycle length, obtained using a detailed model of the $\operatorname{dog} \mathrm{M}$ cell[15] (Fig. 3). Furthermore, the force-calcium relationship used was adapted from the model published by Hunter et al to generate the simulation in this study. 


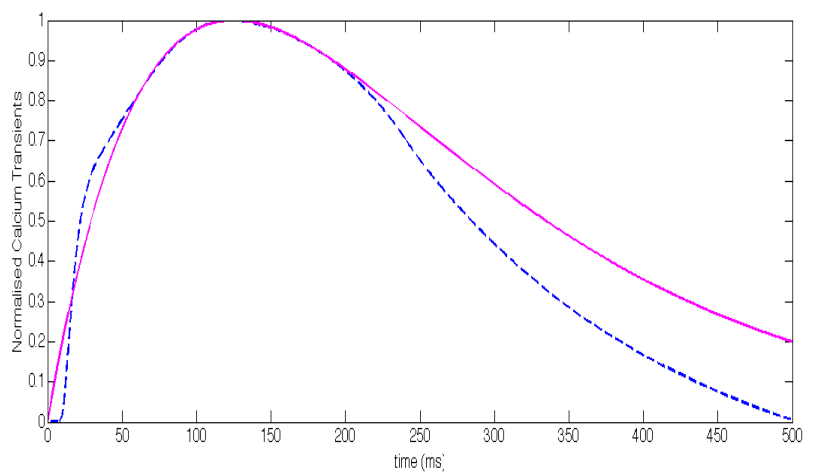

Fig. 3. Normalised Calcium Transients. Solid line indicates the Calcium Transient function used in the simulations. The dotted line indicates the calcium transient obtained from a detailed single $\mathrm{M}$ cell model of a dog.

\subsection{Boundary Conditions}

A left ventricular pressure waveform was provided for this study. The pressure was applied normal to the endocardial walls throughout the cardiac cycle. The basal nodes were fixed to prevent them from moving in the longitudinal direction, but were allowed to move freely in the crossectional direction. The basic cycle length was $500 \mathrm{~ms}$. The timings of the pressure waveform protocol were synchronized with the pacing stimuli of the electromechanical problem, since no hemodynamic model exists in our implementation at the moment (Fig. 4).

\subsection{Simulation protocol}

The simulation protocol follows the time sequence in figure 4. Briefly, the simulations initiated from the zero-pressure configuration, and were allowed to reach steady state before the initiation of the protocol. The mesh is inflated to the pressure in diastasis. The course of the cardiac cycle initiates at that point. The mesh is allowed to passively inflate up to the pressure in end diastole. Slightly before the end of diastole, the electrical stimuli are triggered (at $95 \mathrm{~ms}$ ). The stimulation protocol includes a simulation where all the myocardium is activated synchronously, and using endocardial stimuli (see Table 1).

Table 1. Simulations

\begin{tabular}{|c|c|c|c|}
\hline Number & fibres & Synchronous EP Stimuli & EP Model \\
\hline 1 & DTI fibres & All myocardium & FHN \\
\hline 2 & Streeter fibres & Endocardial & FHN \\
\hline 3 & DTI fibres & Endocardial & FHN \\
\hline
\end{tabular}




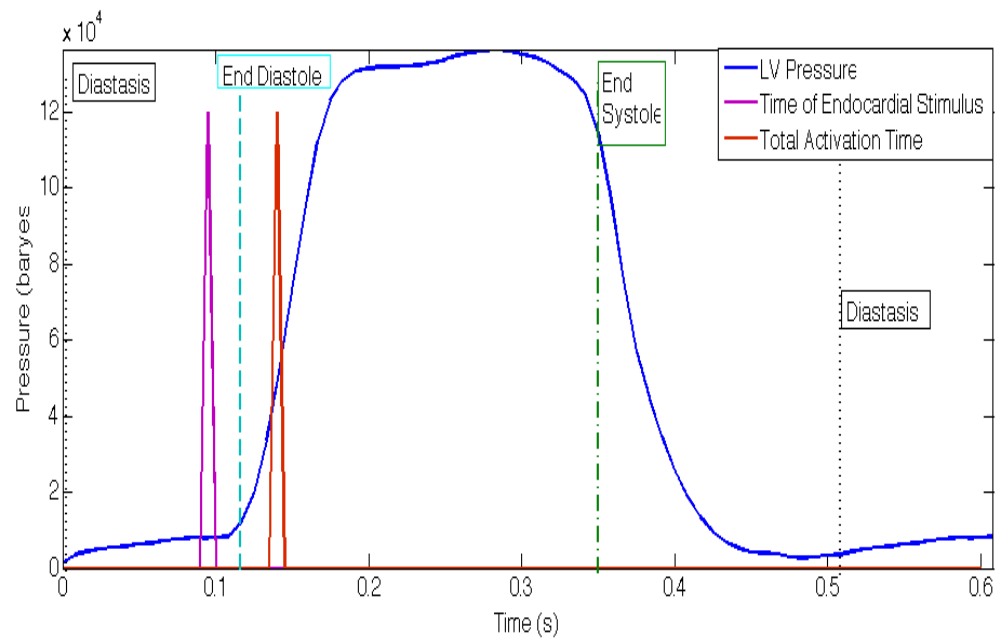

Fig. 4. Pressure time course as boundary conditions normal to the endocardium. Marked are the three defined cardiac cycle hallmarks: Diastasis in black, End Diastole in cyan and End Systole in green. Time of the start of the electrical stimuli is shown in magenta while the total time of activation is shown in orange.

\section{$3 \quad$ Results}

\subsection{Electrical activation: whole vs partial endocardial activation}

Assuming that the electrical activation occurs simultaneously throughout the whole myocardium creates a non-physiological timing of the events on the cardiac cycle. Peak of contraction occurs $90 \mathrm{~ms}$ after electrical activation, which corresponds to a just a few milliseconds after valve opening. For this reason, the use of simultaneous stimuli throughout the myocardium was deemed nonphysiological for our methodology. In the other hand, synchronizing the electromechanic events to the hemodynamics, creates more physiological responses.

\subsection{Fibre orientation: Streeter vs. DTI}

DT-MRI is not always available for implementing electro-mechanical simulations, particularly when creating patient-specific simulations. One of the most popular ways to generate a fibre field is to apply the transmural dispersion as measured experimentally. One of such models was proposed by D. Streeter. The purpose of these simulations is to quantify the influence of the fibre orientation to the electro-mechanics of each model and to verify the error when compared with ground truth data. An important observation, is that the predicted zeropressure configuration of the same geometry was influenced by the fibre field used for each of the simulations. 


\section{Conclusions}

Establishing a more physiological electrical activation sequence provides more realistic timings of the mechanic and hemodynamic events in the cardiac cycle. fibre orientation swiftly modifies the electric activation sequence and the breakthrough of the electric propagation, however more data would be necessary to verify the electrophysiology. Fibre orientation also has an impact on the zero-pressure configuration of the model. Zero-pressure volumes differ depending on the fibres employed, which will have an impact on the overall parameter estimation and the geometrical deformation of the LV.

\section{Acknowledgements}

This work has been done with the support of the grant SEV-2011-00067 of Severo Ochoa Program, awarded by the Spanish Government to the Barcelona Supercomputing Center. Part of the research leading to these results has received funding from the Seventh Framework Programme (FP7/2007-2013) under grant agreement $\mathrm{n}$ 611823. It has also been partially funded from the by the Spanish Ministry of Economy and Competitiveness (TIN2011-28067).

\section{References}

1. Sermesant, M., Moireau, P., Camara, O., Sainte-Marie, J., et al.: Cardiac function estimation from mri using a heart model and data assimilation: advances and difficulties. Medical Image Analysis 10(4) (aug 2006) 642-656

2. Kerckhoffs, R., McCulloch, A., Omens, J., Mulligan, L.: Effects of biventricular pacing and scar size in a computational model of the failing heart with left bundle branch block. Medical Image Analysis 13(2) (2009) 362-369

3. Trayanova, N., Constantino, J., Gurev, V.: Electromechanical models of the ventricles. American Journal of Physiology 301 (2011) 279-286

4. Niederer, S., Kerfoot, E., Benson, A., Bernabeu, M., et al.: Constitutive modelling of passive my- ocardium: a structurally based framework for material characterization. Philosophical Transactions of the Royal Society A: Mathematical, Physical and Engineering Sciences 369(1954) (2009) 4331-4351

5. Camara, O., Sermesant, M., Lamata, P., Wang, L., et al.: Inter-model consistency and complementarity: Learning from ex-vivo imaging and electrophysiological data towards an integrated understanding of cardiac physiology. Progress in Biophysics and Molecular Biology 107(1) (2011) 122-133

6. Tobon-Gomez, C., Craene, M.D., McLeod, K., Tautz, L., et al.: Benchmarking framework for myocardial tracking and deformation algorithms: An open access database. Medical Image Analysis 17(6) (2013) 632-648

7. Wang, V., Lam, H., Ennis, D., Cowan, B., Young, A., Nash, M.: Modelling passive diastolic mechanics with quantitative MRI of cardiac structure and function. Med Image Anal 13(5) (2009) 773-784

8. Streeter, D.: Gross morphology and fibrous structure of the heart. In: Handbook of Physiology: The Cardiovascular System. Volume 1. Oxford University Press (1979) 61-112 
9. Vázquez, M., Arís, R., Houzeaux, G., Aubry, R., et al.: A massively parallel computational electrophysiology model of the heart. International Journal for Numerical Methods in Biomedical Engineering 27(12) (2011) 1991-1929

10. Fitzhugh, R.: Impulses and physiological states in theoretical models of nerve membrane. Biophysical Journal 1 (1961) 445-466

11. ten Tusscher, K.H.W.J., Panfilov, A.V.: Alternans and spiral breakup in a human ventricular tissue model. Am J Physiol Heart Circ Physiol 291(3) (Sep 2006) H1088-H1100

12. Holzapfel, G., Ogden, R.: Constitutive modelling of passive myocardium: a structurally based framework for material characterization. Philosophical Transactions of the Royal Society A: Mathematical, Physical and Engineering Sciences 367(1902) (2009) 3445-3475

13. P. Lafortune, R. Arís, M.V., Houzeaux, G.: Coupled electromechanical model of the heart: Parallel finite element formulation. International Journal for Numerical Methods in Biomedical Engineering 28 (2012) 72-86

14. Hunter, P., McCulloch, A., ter Keurs, H.: Modelling the mechanical properties of cardiac muscle. Progress in Biophysics and Molecular Biology 69(2) (1998) 289-331

15. Flaim, S.N, Giles, W.R., and McCulloch, A.D.: Contributions of sustained INa and IKv4.3 to transmural heterogeneity of early repolarization and arrhythmogenesis in canine left ventricular myocytes. Am J Physiol Heart Circ Physiol 291 (2006) H2617-H2629 